\title{
The use of lesion-specific calcium morphology to guide the appropriate use of dynamic CT myocardial perfusion imaging and CT fractional flow reserve
}

\author{
Xu Dai ${ }^{1 \#}$, Zhigang $\mathrm{Lu}^{2 \#}$, Yarong $\mathrm{Yu}^{3}$, Lihua $\mathrm{Yu}^{3}$, Hao Xu ${ }^{4}$, Jiayin Zhang ${ }^{3}$ \\ ${ }^{1}$ Institute of Diagnostic and Interventional Radiology, Shanghai Jiao Tong University Affiliated Sixth People's Hospital, Shanghai, China; \\ ${ }^{2}$ Department of Cardiology, Shanghai Jiao Tong University Affiliated Sixth People's Hospital, Shanghai, China; ${ }^{3}$ Department of Radiology, Shanghai \\ General Hospital, Shanghai Jiao Tong University School of Medicine, Shanghai, China; ${ }^{4}$ Department of Cardiology, Shanghai General Hospital, \\ Shanghai Jiao Tong University School of Medicine, Shanghai, China
}

Contributions: (I) Conception and design: J Zhang; (II) Administrative support: J Zhang; (III) Provision of study materials or patients: X Dai, Z Lu, Y Yu, H Xu; (IV) Collection and assembly of data: X Dai, Y Yu, L Yu; (V) Data analysis and interpretation: X Dai, L Yu; (VI) Manuscript writing: All authors; (VII) Final approval of manuscript: All authors.

\#These authors contributed equally to this work.

Correspondence to: Jiayin Zhang. Department of Radiology, Shanghai General Hospital, Shanghai Jiao Tong University School of Medicine, 85 Wujin Rd, Shanghai 200080, China. Email: andrewssmu@msn.com; Hao Xu. Department of Cardiology, Shanghai General Hospital, Shanghai Jiao Tong University School of Medicine, 85 Wujin Rd, Shanghai 200080, China. Email: wcnttyyy@163.com.

Background: We aimed to optimize the diagnostic strategy for dynamic computed tomography myocardial perfusion imaging (CT-MPI) and CT fractional flow reserve (CT-FFR) in the evaluation of coronary artery disease (CAD).

Methods: Patients who had undergone coronary CT angiography (CCTA) + dynamic CT-MPI and invasive coronary angiography (ICA)/FFR within a 4-week period were retrospectively included. Lesionspecific characteristics were recorded, and multivariate logistic regression was performed to determine the predictors of mismatched CT findings with ICA results. An optimized diagnostic strategy was proposed based on the diagnostic performance of dynamic CT-MPI and CT-FFR compared with ICA/FFR. A net reclassification index (NRI) was calculated to determine the incremental discriminatory power of optimized CT-FFR + dynamic CT-MPI strategy compared to CT-FFR alone.

Results: The study included 180 patients with 229 diseased vessels. For CT-FFR, a calcified lesion with a calcium arc $>180^{\circ}$ was the only independent predictor for misdiagnosis of ischemic coronary stenosis (odds ratio $=2.367 ; \mathrm{P}=0.002$ ). For noncalcified lesions and calcified lesions with a calcium arc $\leq 180^{\circ}$, the sensitivity and negative predictive value (NPV) of CT-FFR were similar to those of CT-MPI (all P values $>0.05$ ), whereas the specificity and positive predictive value (PPV) of CT-FFR were significantly lower (all P values $<0.05$ ). For calcified lesions with a calcium arc $>180^{\circ}$, the specificity, NPV, and PPV of CT-FFR were inferior to those of CT-MPI (21.2\% vs. $100 \%$, $58.3 \%$ vs. $86.8 \%$, and $62.9 \%$ vs. $100 \%$, respectively; all $\mathrm{P}$ values $<0.05)$. As guided by lesion-specific calcium morphology, an optimized CT-FFR + dynamic CT-MPI strategy $(\mathrm{NRI}=0.2 ; \mathrm{P}=0.004)$ would have resulted in a $27.0 \%$ and $33.9 \%$ reduction of radiation dose and contrast medium consumption, respectively, and $25.3 \%$ of patients would have avoided unnecessary invasive tests.

Conclusions: The diagnostic performance of CT-FFR was significantly inferior in lesions with a calcium arc $>180^{\circ}$. Lesion-specific calcium morphology is the preferred parameter to guide the appropriate use of CT-based functional assessment. 


\begin{abstract}
Keywords: Coronary artery disease (CAD); fractional flow reserve (FFR); myocardial perfusion imaging (MPI);
\end{abstract} computed tomography (CT); myocardial blood flow (MBF)

Submitted May 07, 2021. Accepted for publication Sep 18, 2021.

doi: $10.21037 /$ qims-21-491

View this article at: https://dx.doi.org/10.21037/qims-21-491

\section{Introduction}

The functional evaluation of the hemodynamic significance of coronary stenosis plays a vital role in optimizing the treatment strategy and results in more favorable clinical outcomes $(1,2)$. Due to recent advances in technology, computed tomography (CT) now enables functional assessment of coronary artery disease (CAD) in two ways: dynamic computed tomography myocardial perfusion imaging (CT-MPI) and CT fractional flow reserve (CTFFR) (3-9).

Dynamic CT-MPI allows for absolute quantification of myocardial blood flow (MBF) and is highly accurate in diagnosing ischemic coronary stenosis compared with invasive FFR $(10,11)$. However, it has the disadvantage of additional image acquisition and use of vasodilators, which result in a relatively high radiation dose and contrast medium consumption. In contrast to dynamic CT-MPI, CT-FFR [either based on the computational fluid dynamic (CFD) method or the machine learning (ML) approach] can simulate a lesion-specific FFR value using conventional coronary CT angiography (CCTA) data. However, its clinical value could be limited in cases with diffusely calcified lesions or compromised image quality (12).

In light of the above facts, it is clinically relevant to investigate the impact of the lesion-specific characteristics of coronary stenosis—such as the calcium burden, calcium morphology, and stenotic extent-on the diagnostic accuracy of dynamic CT-MPI and CT-FFR. The determination of specific lesion characteristics that can best predict the appropriate use of CT-FFR or dynamic CT-MPI is expected to optimize the application of CTbased functional imaging methods with less radiation and a preserved diagnostic performance. Therefore, the primary aim of this study was to test the hypothesis that specific lesion-specific characteristics would impact the diagnostic performance and optimize the selective use of CT-FFR and dynamic CT-MPI. We present the following article in accordance with the STARD reporting checklist (available at https://dx.doi.org/10.21037/qims-21-491).

\section{Methods}

\section{Patient population}

This study was conducted in accordance with the Declaration of Helsinki (as revised in 2013). The hospital's ethic committee approved this retrospective study (No. 2020-91) and the waiver for written informed consent from all patients. Between January 1, 2017, and December 31, 2020, we retrospectively reviewed and included all patients who had undergone dynamic CT-MPI + CCTA and invasive coronary angiography (ICA)/FFR within a 4-week period. The indication for dynamic CT-MPI + CCTA was to diagnose hemodynamically significant $\mathrm{CAD}$ in patients with stable chest pain and an intermediate-to-high pretest probability of CAD according to the updated DiamondForrester score (pretest probability $\geq 15 \%$ ). The exclusion criteria were as follows: (I) patients with a previous history of coronary revascularization; (II) patients with a previous history of myocardial infarction; (III) patients with suspected cardiomyopathy or microvascular dysfunction; (IV) a significantly impaired image quality on dynamic CTMPI or CCTA.

\section{Dynamic CT-MPI + CCTA acquisition}

A third-generation dual-source CT (SOMATOM Force, Siemens Healthineers, Erlangen, Germany) was used for the image acquisition. The scan was divided into 3 steps: the calcium score, dynamic CT-MPI, and CCTA. In brief, a coronary Agatston calcium score (CACS) was initially acquired to calculate the calcification burden of each epicardial vessel. An intravenous adenosine triphosphate (ATP) infusion was maintained for $3 \mathrm{~min}$ at $160 \mu \mathrm{g} / \mathrm{kg} / \mathrm{min}$ before the CT-MPI scan. Dynamic acquisition was set at the end-systolic phase (triggered at $250 \mathrm{~ms}$ after the $\mathrm{R}$ wave in all patients) and started at $4 \mathrm{~s}$ after onset of the contrast injection. Scans were launched every second or third heart cycle according to the patient's heart rate with use of a shuttle mode technique. The reference tube voltage and effective current was $80 \mathrm{kVp}$ and $300 \mathrm{mAs}$, respectively, 
with CARE $\mathrm{kV}$ and CARE dose 4D (Siemens Healthineers, Erlangen, Germany) being used to reduce the radiation dose.

The CCTA acquisition was performed 5 min after the dynamic CT-MPI with nitroglycerin given sublingually in all participants. The detailed parameters of the contrast medium injection, dynamic CT-MPI, and CCTA acquisition are provided in the Appendix 1.

\section{Image analysis of CCTA}

A smooth kernel ( $\mathrm{Bv} 40)$ and third-generation iterative reconstruction technique (strength 3, ADMIRE, Siemens Healthineers) were used for the CCTA reconstruction. The best systolic and diastolic images were transferred to a commercially available workstation (SyngoVia VB 2.0, Siemens Healthineers) for further analysis. All lesions on the major epicardial arteries (diameter $\geq 2 \mathrm{~mm}$ ) were evaluated with dedicated plaque analysis software (Coronary Plaque Analysis version 4.3, Siemens Healthineers). The following qualitative and quantitative parameters were measured and recorded: (I) diameter of the stenosis (DS) and lesion length; (II) patient-based and vessel-based CACS; (III) lesion-specific calcium morphology according to the involved quadrants by calcification (13). The detailed definitions of the above parameters are given in the Appendix 1. The Coronary Artery Disease-Reporting and Data System (CAD-RADS) was used for the patient-based analysis regarding the extent of stenosis (14).

Two cardiovascular radiologists (with 12 years and 4 years of experience in cardiac imaging) independently measured and evaluated all the above parameters. The mean of all measured values was adopted for the analysis.

\section{Image analysis of CT-MPI}

The CT-MPI images were reconstructed using a dedicated kernel (Qr36) to reduce iodine beam-hardening artifacts and were analyzed using a CT-MPI software package (myocardial perfusion analysis, VPCT body, Siemens Healthineers). Motion correction was manually applied to correct for breathing-related misregistration of the left ventricle. A hybrid deconvolution model was employed to quantify MBF, as previously reported (15).

For the quantitative analysis, absolute MBF was measured on the segment base by drawing a region of interest (ROI) on the short axis view of the left ventricle covering the whole myocardial segment (with the exclusion of the endocardial and epicardial interface) (10) according to the 17-segment model with the exclusion of the apical segment (16). The mean MBF of segments with upstream vessel stenosis $\geq 30 \%$ were recorded. According to a previous study, $\mathrm{MBF}<99 \mathrm{~mL} / \mathrm{min} / 100 \mathrm{~mL}$ was considered to indicate the presence of myocardial ischemia (10). MBF was independently analyzed by 2 cardiovascular radiologists (with 12 years and 4 years of experience in cardiac imaging) who were blinded to the clinical information and invasive FFR results. The mean of all measured values was adopted for the analysis.

\section{Image analysis of CT-FFR}

The current study used an ML-based approach for the CTFFR simulation (cFFR, version 3.0, Siemens Healthineers). In brief, this model was trained on a large database of synthesized coronary anatomies, where the reference values were computed using a CFD-based model (17). The details regarding how this ML-based model was trained and how on-site processing was performed are given in the Appendix 1. The lesion-specific CT-FFR values were measured at 1 to 2 $\mathrm{cm}$ distal to the lesion. CT-FFR was independently analyzed by the same 2 aforementioned cardiovascular radiologists, and the mean values of measurements were used for further analysis.

\section{ICA and FFR measurement}

ICA was performed and assessed by 2 interventional cardiologists (with 26 years and 20 years of experience in coronary intervention). At least 2 views were obtained for each major vessel. The stenotic extent of each lesion was visually evaluated and recorded. All lesions with a stenotic extent from $30 \%$ to $90 \%$ were referred for invasive FFR measurement using a previously described method (18). Hyperemia was induced by an intravenous infusion of ATP at $160 \mu \mathrm{g} / \mathrm{kg} / \mathrm{min}$. Lesions with an FFR value $\leq 0.8$ were considered physiologically significant. In addition, lesions with more than $90 \%$ stenosis were considered ischemic $(19,20)$ and directly referred for revascularization without FFR measurement. Lesions with less than 30\% stenosis were considered nonischemic and treated conservatively.

\section{Comparison of CT-MPI and CT-FFR with ICA and invasive FFR}

For the prespecified reference standard, all lesions were 
classified as ischemic or nonischemic according to the ICA/ FFR findings. Lesions with an invasive FFR value $\leq 0.8$ or a stenotic extent $\geq 90 \%$ were deemed ischemic, whereas lesions with an invasive FFR $>0.8$ or a stenotic extent $\leq 30 \%$ were considered nonischemic. Similarly, vessels causing ischemia were defined as arteries with at least one ischemic stenosis, whereas vessels not causing ischemia were defined as arteries without any ischemic stenosis.

A comparison of various CT-derived parameters with the reference standard was made on a vessel-based and patient-based analysis. For CT-FFR, the lesion with the smallest CT-FFR value on 1 vessel (or the most distal lesion where tandem lesions were present) was chosen for further analysis. For CT-MPI-derived MBF, the mean values of the stenosis-subtended segments were used for further comparisons. Chronic total occlusions were not included in the analysis since neither CT-FFR nor invasive FFR could measure these.

\section{Statistical analysis}

Continuous variables are presented as the mean $\pm \mathrm{SD}$ for normally distributed data or the median and interquartile range for nonnormally distributed data. Categorical variables are presented as numbers and percentages. Groups and subgroups were compared with the Student's $t$ test or Wilcoxon rank-sum test for continuous variables and the chi-square test or Fisher's exact test for categorical variables. Univariate and multivariate logistic regressions were performed to determine the impact of specific factors on the mismatch of MBF and CT-FFR with ICA results by adjusting for characteristics of interest. The per-patient and per-vessel areas under the curve (AUCs) derived from the receiver operating characteristic (ROC) curve analysis were calculated using invasive FFR as the reference standard. The diagnostic performance of the test strategy was also compared between subgroups, and the DeLong test was used to compare AUCs (21). The optimal threshold of CTFFR and MBF to determine the hemodynamic significance of coronary stenosis was calculated using the Youden index. For all patients, per-vessel and per-patient sensitivity, specificity, positive predictive value (PPV), negative predictive value (NPV), and the diagnostic accuracy of $\mathrm{MBF}$ and CT-FFR were calculated with $95 \%$ confidence intervals (CIs). The McNemar test was used to compare sensitivities and specificities, and the chi-square test was used for dependent proportions across subgroups. The net reclassification index (NRI) was calculated to determine the incremental discriminatory power of optimized CTFFR + dynamic CT-MPI strategy compared with CT-FFR alone (22). Intraobserver and interobserver agreements were analyzed with a concordance correlation coefficient. A $\mathrm{P}$ value $<0.05$ was considered statistically significant. All statistical analyses were performed with SPSS version 23 (IBM Corporation, Armonk, NY, USA) and MedCalc version 19.0.4 (MedCalc Software, Ostend, Belgium) software.

\section{Results}

\section{Patient characteristics}

A total of 394 patients with chest pain who were referred for dynamic CT-MPI + CCTA between January 1, 2017, and December 31, 2020, were retrospectively reviewed. Overall, 214 patients were excluded due to various reasons (as shown in Figure 1), and 21 patients were excluded because of suspected cardiomyopathy $(8 / 21)$ or microvascular dysfunction (13/21). In addition, 95 patients with an absence of hemodynamically significant stenosis, as well as 8 patients with more than a 4-week interval between the CT examination and ICA/FFR, were further excluded. Finally, 180 patients with 229 diseased vessels were included in the current study with a median time interval of 7 days between the CT examination and the ICA/FFR measurement. The mean cumulative effective dose for dynamic CTMPI + CCTA was 5.6 (range, 4.7-7.0) mSv. The detailed demographic data and lesion characteristics are shown in Tables 1,2. Good intraobserver and interobserver agreement was found for measurements of all parameters (Tables S1,S2).

\section{Predictors of mismatched CT functional test results with reference to ICA/FFR}

Various lesion-specific parameters were included in the univariate and multivariate analyses to determine the predictors of mismatched results between the CT functional tests and ICA/FFR. For CT-FFR, calcium morphology was the only independent predictor of the misdiagnosis of ischemic coronary stenosis (odds ratio $=2.367 ; \mathrm{P}=0.002$; Table S3 and Figure 2). Other features, such as CACS, DS, and lesion length, did not impact the diagnostic accuracy of CT-FFR. For dynamic CT-MPI, none of the lesion-specific characteristics demonstrated a predictive value for the misdiagnosis of the hemodynamic significance of coronary 


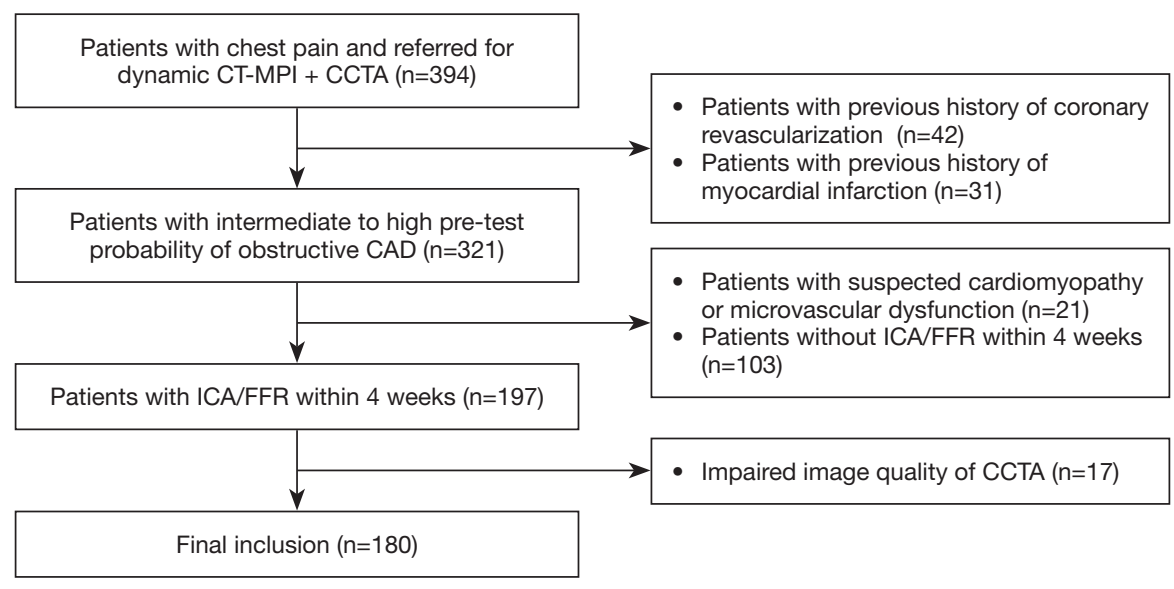

Figure 1 Flow chart of inclusion and exclusion criteria. CT-MPI, computed tomography myocardial perfusion imaging; CCTA, coronary computed tomography angiography; CAD, coronary artery disease; ICA, invasive coronary angiography; FFR, fractional flow reserve.

Table 1 Demographic data

\begin{tabular}{|c|c|}
\hline Characteristics & Value $(n=180)$ \\
\hline Male, n (\%) & $137(76.1)$ \\
\hline Age, years & $65.4 \pm 9.8$ \\
\hline BMI & $24.21 \pm 3.04$ \\
\hline \multicolumn{2}{|l|}{ High-risk factors, n (\%) } \\
\hline Hypertension & $129(71.6)$ \\
\hline DM & $88(48.8)$ \\
\hline Dyslipidemia & $51(28.3)$ \\
\hline Smoking & $77(42.8)$ \\
\hline \multicolumn{2}{|l|}{ CAD-RADS, n (\%) } \\
\hline 2 & $16(8.9)$ \\
\hline 3 & $44(24.4)$ \\
\hline $4 \mathrm{~A}$ & $120(66.7)$ \\
\hline \multicolumn{2}{|l|}{ Angina, n (\%) } \\
\hline CCS class I & $8(4.4)$ \\
\hline CCS class II & $91(50.6)$ \\
\hline CCS class III & $67(37.2)$ \\
\hline CCS class IV & $14(7.8)$ \\
\hline $\begin{array}{l}\text { CT-MPI + CCTA radiation dose, mSv, median } \\
\text { (IQR) }\end{array}$ & $5.6(4.7-7.0)$ \\
\hline CT-MPI radiation dose, mSv, median (IQR) & $2.9(2.4-3.7)$ \\
\hline Stress HR/Baseline HR, median (IQR) & $1.19(1.12-1.29)$ \\
\hline \multicolumn{2}{|c|}{$\begin{array}{l}\text { BMI, body mass index; DM, diabetes mellitus; CAD-RADS, } \\
\text { Coronary Artery Disease-Reporting and Data System; CCS, } \\
\text { Canadian Cardiovascular Society; CT, computed tomography; } \\
\text { MPI, myocardial perfusion imaging; CCTA, coronary computed } \\
\text { tomography angiography; HR, heart rate. }\end{array}$} \\
\hline
\end{tabular}

Table 2 Lesion characteristics

\begin{tabular}{lc}
\hline Characteristics & Value $(\mathrm{n}=229)$ \\
\hline Hemodynamically significant, $\mathrm{n}(\%)$ & $92(40.1)$ \\
Invasive FFR $<0.8, \mathrm{n}(\%)$ & $42(18.3)$ \\
Diameter stenosis $\geq 90 \%, \mathrm{n}(\%)$ & $50(21.8)$ \\
Calcified segments of target vessels, number, $\mathrm{n}(\%)^{\star}$ & $83(36.2)$ \\
0 & $59(25.8)$ \\
1 & $56(24.5)$ \\
2 & $22(9.6)$ \\
3 & $7(3.1)$ \\
4 & $2(0.8)$ \\
5 & \\
Calcium morphology, number, $\mathrm{n}(\%)$ & $89(38.9)$ \\
Noncalcified lesions & $58(25.3)$ \\
$0^{\circ}<$ calcification arc $\leq 180^{\circ}$ & $82(35.8)$ \\
$180^{\circ}<$ calcification arc $\leq 360^{\circ}$ & $67 \pm 17$ \\
Diameter stenosis, \%, mean $\pm \mathrm{SD}$ & $109.0(88.1-146.0)$ \\
Patient-based CACS, median (IQR) & $0.75(0.64-0.83)$ \\
Vessel-based CACS, median (IQR) & $239.0(54.3-748.6)$ \\
Lesion length, mm, median $(\mathrm{IQR})$ & $103.9(10.8-336.1)$ \\
MBF, mL/100 mL/min, median (IQR) & $25.4(16.9-39.0)$ \\
\hline$* F R$, median $(I \mathrm{Q})$ & \\
\hline
\end{tabular}

*, defined as the number of target vessel segments having wall calcification, according to the 18-segment model by the Society of Cardiovascular Computed tomography. Branch vessel segments were included when target lesions were located upstream. FFR, fractional flow reserve; CACS, coronary artery calcium score; MBF, myocardial blood; flow CT, computed tomography. 



Figure 2 Representative cases of CT-FFR and dynamic CT-MPI evaluations compared with ICA/FFR. (A) CCTA shows a calcified lesion at the middle LCx with moderate stenosis and a calcium arc less than $180^{\circ}$ in a 76 -year-old female. ICA confirms moderate stenosis at the middle LCx, which is hemodynamically insignificant as revealed by FFR. CT-FFR simulation and dynamic CT-MPI findings are both consistent with FFR results. (B) CCTA shows a diffusely calcified lesion at the middle LAD with severe stenosis and a calcium arc of more than $180^{\circ}$ in a 53 -year-old male. ICA reveals moderate stenosis at the middle LAD, which is hemodynamically insignificant as validated by FFR. CT-FFR simulation misdiagnoses the lesion as hemodynamically significant, whereas dynamic CT-MPI makes the correct diagnosis. ICA, invasive coronary angiography; FFR, fractional flow reserve; CT-MPI, computed tomography myocardial perfusion imaging; CCTA, coronary computed tomography angiography; LCx, left circumflex; LAD, left anterior descending; MBF, myocardial blood flow.

stenosis (Table S3).

\section{Diagnostic performance of CT functional tests according to lesion-specific characteristics}

When stratified by calcium morphology, the diagnostic performance of $\mathrm{MBF}$ showed no significant difference across subgroups (AUC $=0.928,95 \%$ CI: 0.853-0.972; 0.988, 95\% CI: 0.917-1.000; and 0.942, 95\% CI: 0.868-0.982); all $\mathrm{P}$ values $>0.05)$. However, the AUC value for CT-FFR in the group with $>180^{\circ}$ calcification significantly decreased when compared with the other 2 subgroups $(0.643,95 \%$ CI: $0.534-0.749$ vs. 0.862 , $95 \%$ CI: $0.773-0.926$; 0.643 , $95 \%$ CI: $0.534-0.749$ vs. 0.883 , $95 \%$ CI: $0.772-0.953$; P value $=0.004$ and 0.002 , respectively). In addition, CTFFR and MBF had a similar diagnostic performance among subgroups stratified by vessel-based CACS (Figure 3).
Using the best cutoff value of $\leq 99 \mathrm{~mL} / 100 \mathrm{~mL} / \mathrm{min}$, the diagnostic accuracy of MBF in the subgroup analysis according to calcium morphology was $92.1 \%$ (95\% CI: $84.5-96.8 \%$ ), $96.6 \%$ (95\% CI: $88.1-99.6 \%$ ), and $93.9 \%$ (95\% CI: 86.3-98.0\%), respectively, whereas these values for ML-based CT-FFR (when using the cutoff value of $\leq 0.78$ derived from the ROC curve analysis) were $82.0 \%$ (95\% CI: $72.5-89.4 \%$ ), $74.1 \%$ (95\% CI: 61.0-84.7\%), and 64.6\% (95\% CI: $53.3-74.9 \%$ ) (Table 3). As shown by the vessel-based analysis stratified by calcium morphology, MBF outperformed CT-FFR in terms of accuracy, specificity, and PPV, but there was no significant difference between MBF and CT-FFR regarding sensitivity and NPV (Table 4 and Figure 4) for noncalcified lesions and calcified lesions with a calcium arc $\leq 180^{\circ}$. In terms of the overall diagnostic performance as stratified by calcium morphology, there was no significant difference between 



Figure 3 ROC curve analysis of dynamic CT-MPI and CT-FFR for identifying functionally significant coronary stenosis according to calcium morphology and vessel-based CACS compared with ICA/FFR. (A,B) When stratified by calcium morphology and vessel-based CACS, dynamic CT-MPI maintains a similar AUC across different subgroups (all P values $>0.05$ ). (C) The AUC of CT-FFR for lesions with $>180^{\circ}$ calcium arc is significantly smaller than that of the other 2 subgroups (all $\mathrm{P}$ values $<0.05$ ). (D) When stratified by vessel-based CACS, the AUCs of CT-FFR are not statistically different across the different subgroups (all P values $>0.05$ ). AUC, area under curve; CACS, coronary artery calcium score; CT, computed tomography; CT-MPI, computed tomography myocardial perfusion imaging; FFR, fractional flow reserve; ICA, invasive coronary angiography; MBF, myocardial blood flow; ROC, receiver operating characteristic.

Table 3 Univariate and multivariate analyses of CT-FFR for mismatch with ICA/FFR

\begin{tabular}{|c|c|c|c|c|c|c|}
\hline Characteristics & \multicolumn{3}{|c|}{ Univariate analysis } & \multicolumn{3}{|c|}{ Multivariate analysis } \\
\hline Vessel-based CACS & 1.001 & $1.000-1.001$ & 0.227 & 1.000 & $0.999-1.002$ & 0.544 \\
\hline Diameter stenosis & 0.926 & $0.778-1.102$ & 0.385 & 0.852 & $0.703-1.034$ & 0.104 \\
\hline Lesion length & 1.005 & $0.988-1.021$ & 0.579 & - & - & - \\
\hline Number of calcified segments of target vessels & 1.058 & $0.803-1.395$ & 0.689 & 0.633 & $0.386-1.040$ & 0.071 \\
\hline
\end{tabular}

$\mathrm{CT}$, computed tomography; FFR, fractional flow reserve; ICA, invasive coronary angiography; CACS, coronary artery calcium score; Cl, confidence interval; OR, odds ratio. 
Table 4 Vessel-based diagnostic performance of CT-FFR and MBF according to calcium morphology

\begin{tabular}{|c|c|c|c|c|c|c|c|c|c|}
\hline & \multicolumn{3}{|c|}{ Noncalcified lesions $(n=89)$} & \multicolumn{3}{|c|}{$0^{\circ}<$ calcification $\operatorname{arc} \leq 180^{\circ}(n=58)$} & \multicolumn{3}{|c|}{$180^{\circ}<$ calcification $\operatorname{arc} \leq 360^{\circ}(n=82)$} \\
\hline Sensitivity & $\begin{array}{c}85.2(23 / 27) \\
{[66.3-95.8]}\end{array}$ & $\begin{array}{r}88.9(24 / 27) \\
{[70.4-97.7]}\end{array}$ & 1.000 & $\begin{array}{c}100(16 / 16) \\
{[79.4-100.0]}\end{array}$ & $\begin{array}{c}93.8(15 / 16) \\
{[69.8-99.8]}\end{array}$ & 1.000 & $\begin{array}{c}89.8(44 / 49) \\
{[77.8-96.6]}\end{array}$ & $\begin{array}{c}89.8(44 / 49) \\
{[77.8-96.6]}\end{array}$ & 1.000 \\
\hline Specificity & $\begin{array}{c}95.2(59 / 62) \\
{[86.5-99.0]}\end{array}$ & $\begin{array}{c}79.0(49 / 62) \\
{[66.8-88.3]}\end{array}$ & 0.006 & $\begin{array}{c}95.2(40 / 42) \\
{[83.8-99.4]}\end{array}$ & $\begin{array}{c}66.7(28 / 42) \\
{[50.5-80.4]}\end{array}$ & 0.004 & $\begin{array}{c}100(33 / 33) \\
{[89.4-100]}\end{array}$ & $\begin{array}{l}21.2(7 / 33) \\
{[10.0-38.9]}\end{array}$ & $<0.001$ \\
\hline PPV & $\begin{array}{c}88.5(23 / 26) \\
{[71.5-95.9]}\end{array}$ & $\begin{array}{c}64.9(24 / 37) \\
{[52.8-75.3]}\end{array}$ & 0.043 & $\begin{array}{l}88.9(16 / 18) \\
{[67.4-96.9]}\end{array}$ & $\begin{array}{l}51.7(15 / 29) \\
{[40.7-62.6]}\end{array}$ & 0.009 & $\begin{array}{c}100(44 / 44) \\
-\end{array}$ & $\begin{array}{c}62.9(44 / 70) \\
{[58.1-67.4]}\end{array}$ & $<0.001$ \\
\hline Accuracy & $\begin{array}{c}92.1(82 / 89) \\
{[84.5-96.8]}\end{array}$ & $\begin{array}{c}82.0(73 / 89) \\
{[72.5-89.4]}\end{array}$ & 0.007 & $\begin{array}{c}96.6(56 / 58) \\
{[88.1-99.6]}\end{array}$ & $\begin{array}{c}74.1(43 / 58) \\
{[61.0-84.7]}\end{array}$ & 0.013 & $\begin{array}{c}93.9(77 / 82) \\
{[86.3-98.0]}\end{array}$ & $\begin{array}{c}62.2(51 / 82) \\
{[50.8-72.7]}\end{array}$ & $<0.001$ \\
\hline
\end{tabular}

*, MBF cutoff value $=99 \mathrm{~mL} / 100 \mathrm{~mL} / \mathrm{min} ;{ }^{\S}, \mathrm{CT}-\mathrm{FFR}$ cutoff value $=0.78$. Data are presented as percentage [95\% Cl]. CT, computed tomography; FFR, fractional flow reserve; MBF, myocardial blood flow; NPV, negative predictive value; PPV, positive predictive value.
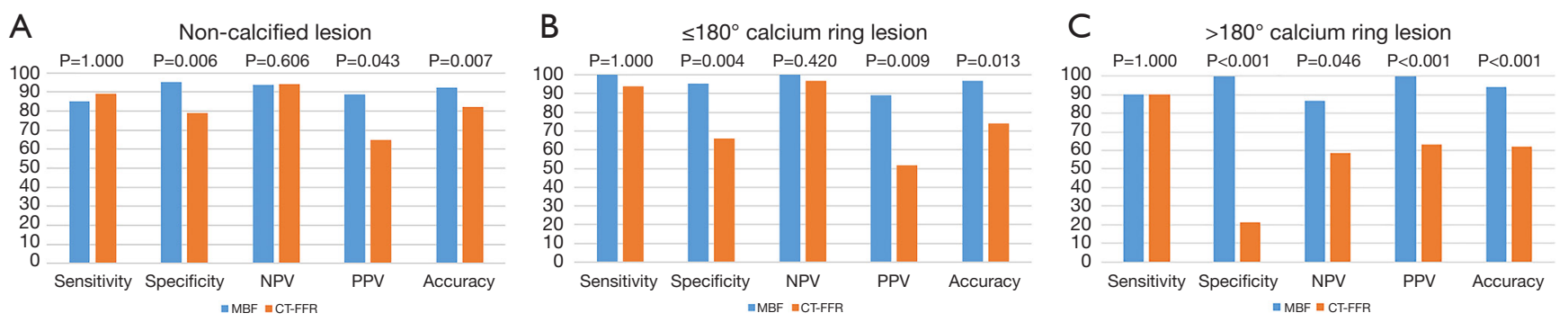

Figure 4 Direct comparison of the diagnostic performance of dynamic CT-MPI and CT-FFR in identifying functionally significant lesions according to calcium morphology. (A,B) For noncalcified lesions and calcified lesions with a calcium arc $\leq 180^{\circ}$, the diagnostic accuracy, specificity, and PPV of dynamic CT-MPI are significantly higher than those of CT-FFR, whereas the sensitivity and NPV are similar for the 2 methods. (C) For calcified lesions with calcium arc $>180^{\circ}$, the diagnostic accuracy, specificity, NPV, and PPV of dynamic CT-MPI are significantly higher than those of CT-FFR, whereas the sensitivity is similar for the 2 methods. CT, computed tomography; CT-MPI, computed tomography myocardial perfusion imaging; FFR, fractional flow reserve; MBF, myocardial blood flow; NPV, negative predictive value; PPV, positive predictive value.

CT-FFR and dynamic CT-MPI for noncalcified lesions as shown by the ROC analysis, whereas significantly impaired performance was observed for CT-FFR for calcified lesions (details shown in Figure S1). The analysis based on vessel-specific CACS also revealed similar results, with MBF outperforming CT-FFR in all subgroups (Table S4). It is also worth noting that when stratified by CADRADS grade (CAD-RADS 2-3 vs. CAD-RADS 4: 78.3\% vs. $75.8 \% ; \mathrm{P}=0.709)$ or patient-based CACS $(0 \leq$ total CACS $<100$ vs. $100 \leq$ total CACS $<400$ vs. total CACS $\geq 400$ : $79.7 \%$ vs. $73.1 \%$ vs. $76.6 \% ; \mathrm{P}=0.704$ ), the diagnostic accuracy of CT-FFR did not show any significant discrepancy between the subgroups (Table S5 and Table 5).
A guide for the appropriate sequential use of CT functional tests and the reclassification benefit

As shown in Table 3, for noncalcified lesions and calcified lesions with a calcium arc $\leq 180^{\circ}$, the sensitivity and NPV of CT-FFR were similar to those of MBF, whereas the specificity and PPV of CT-FFR were significantly impaired. For calcified lesions with a calcium arc $>180^{\circ}$, both the NPV and PPV of CT-FFR were inferior to those of MBF. Therefore, we proposed an alternative sequential use of CT functional tests, as shown in Figure 5.

According to this protocol, if dynamic CT-MPI had been used selectively in those patients with positive CT- 
Table 5 Patient-based diagnostic performance of CT-FFR and MBF according to patient-based CACS

\begin{tabular}{|c|c|c|c|c|c|c|c|c|c|}
\hline & \multicolumn{3}{|c|}{$0 \leq$ total CACS $<100(n=64)$} & \multicolumn{3}{|c|}{$100 \leq$ total CACS $<400(n=52)$} & \multicolumn{3}{|c|}{ Total CACS $\geq 400(n=64)$} \\
\hline Sensitivity & $\begin{array}{l}76.5(13 / 17) \\
{[50.1-93.2]}\end{array}$ & $\begin{array}{c}76.5(13 / 17) \\
{[50.1-93.2]}\end{array}$ & 1.000 & $\begin{array}{c}95.8(23 / 24) \\
{[78.9-100]}\end{array}$ & $\begin{array}{c}83.3(20 / 24) \\
{[62.6-95.3]}\end{array}$ & 0.250 & $\begin{array}{c}91.9(34 / 37) \\
{[78.1-98.3]}\end{array}$ & $\begin{array}{c}94.6(35 / 37) \\
{[81.8-99.3]}\end{array}$ & 1.000 \\
\hline Specificity & $\begin{array}{c}93.6(44 / 47) \\
{[82.5-98.7]}\end{array}$ & $\begin{array}{c}80.9(38 / 47) \\
{[66.7-90.9]}\end{array}$ & 0.001 & $\begin{array}{l}100(28 / 28) \\
{[87.7-100]}\end{array}$ & $\begin{array}{l}64.3(18 / 28) \\
{[44.1-81.4]}\end{array}$ & 0.001 & $\begin{array}{l}100(27 / 27) \\
{[87.2-100]}\end{array}$ & $\begin{array}{l}51.9(14 / 27) \\
{[32.0-71.3]}\end{array}$ & $<0.001$ \\
\hline PPV & $\begin{array}{c}81.3(13 / 16) \\
{[58.4-93.0]}\end{array}$ & $\begin{array}{c}59.1(13 / 22) \\
{[43.1-73.3]}\end{array}$ & 0.147 & $\begin{array}{c}100(23 / 23) \\
-\end{array}$ & $\begin{array}{c}66.7(20 / 30) \\
{[54.1-77.2]}\end{array}$ & 0.003 & $\begin{array}{c}100(34 / 34) \\
-\end{array}$ & $\begin{array}{c}72.9(35 / 48) \\
{[64.4-80.1]}\end{array}$ & 0.001 \\
\hline Accuracy & $\begin{array}{l}89.1(57 / 64) \\
{[78.8-95.5]}\end{array}$ & $\begin{array}{l}79.7(51 / 64) \\
{[67.8-88.7]}\end{array}$ & 0.180 & $\begin{array}{c}98.1(51 / 52) \\
{[89.7-100]}\end{array}$ & $\begin{array}{c}73.1(38 / 52) \\
{[59.0-84.4]}\end{array}$ & $<0.001$ & $\begin{array}{l}95.3(61 / 64) \\
{[86.9-99.0]}\end{array}$ & $\begin{array}{l}76.6(49 / 64) \\
{[64.3-86.3]}\end{array}$ & 0.001 \\
\hline
\end{tabular}

${ }^{*}$, MBF cutoff value $=99 \mathrm{~mL} / 100 \mathrm{~mL} / \mathrm{min} ;{ }^{\S}, \mathrm{CT}-\mathrm{FFR}$ cutoff value $=0.76$. Data are presented as percentage [95\% Cl]. CT, computed tomography; FFR, fractional flow reserve; MBF, myocardial blood flow; CACS, coronary artery calcium score; Cl, confidence interval; OR, odds ratio; NPV, negative predictive value; PPV, positive predictive value.

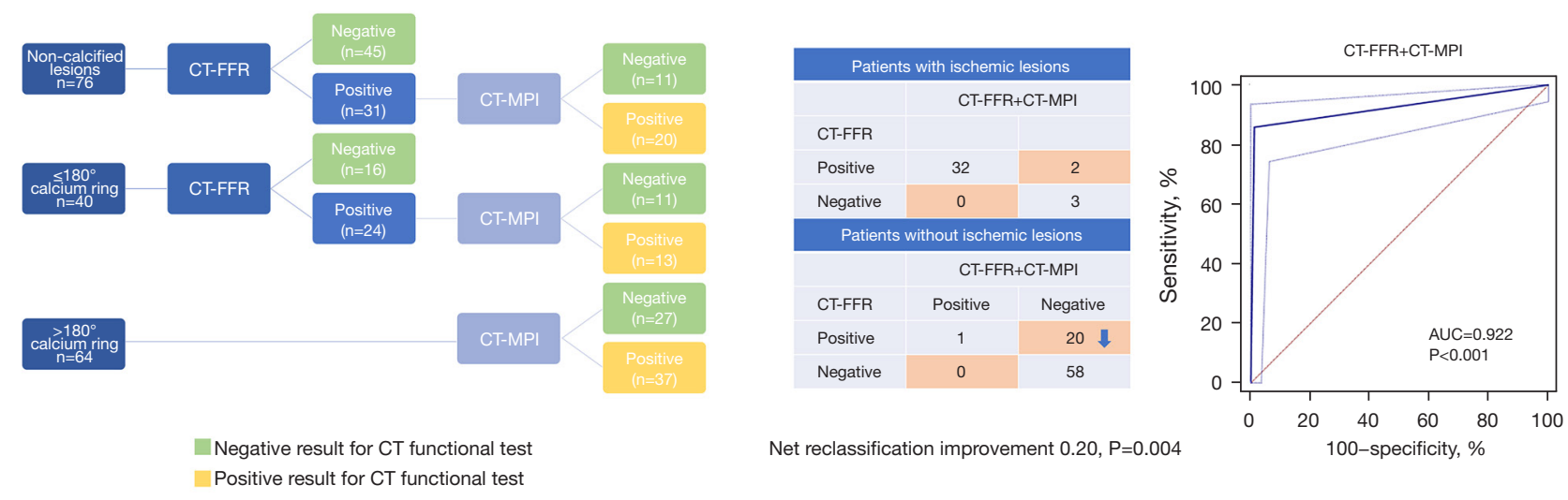

Figure 5 Optimized CT-FFR + dynamic CT-MPI strategy and its net reclassification improvement. For patients with noncalcified lesions or calcified lesions with a calcium arc $\leq 180^{\circ}$, negative CT-FFR results can be used to safely exclude hemodynamically significant stenosis, whereas dynamic CT-MPI can be added for patients with positive CT-FFR results to reduce false-positive cases. For patients with calcified lesions having a calcium arc $>180^{\circ}$, dynamic CT-MPI can be used to replace CT-FFR to minimize both false-positive and false-negative cases. This optimized strategy would allow $25.3 \%$ of patients to avoid unnecessary ICA/FFR, compared to using CT-FFR alone. In addition, instead of performing CT-FFR + dynamic CT-MPI in all patients, the selective usage of dynamic CT-MPI in addition to CT-FFR would reduce the radiation dose by $27.0 \%$ and contrast medium consumption by $33.9 \%$. The net reclassification improvement of this optimized strategy is $0.2(\mathrm{P}=0.004)$ compared to the CT-FFR alone strategy. The AUC of this optimized strategy is 0.922 ( $\mathrm{P}<0.001)$ compared with ICA/FFR. AUC, area under curve; CT, computed tomography; CT-MPI, computed tomography myocardial perfusion imaging; FFR, fractional flow reserve; ICA, invasive coronary angiography.

FFR results who had non-calcified and mild-to-moderately calcified lesions and in all patients with moderate-toseverely calcified lesions, this optimized diagnostic strategy would have saved $33.9 \%(61 / 180)$ of patients from undergoing dynamic CT-MPI. With reference to ICA/FFR, it would also have resulted in a $27.0 \%$ and $33.9 \%$ reduction of radiation dose and contrast medium consumption, respectively, while maintaining the diagnostic performance (Figure 5). Compared to CT-FFR alone, unnecessary downstream invasive tests could have been avoided in 
$25.3 \%$ of reclassified patients with the selective addition of dynamic CT-MPI. The NRI of this optimized CT-FFR + dynamic CT-MPI strategy over CT-FFR alone was 0.2 $(\mathrm{P}=0.004)$.

\section{Discussion}

The major findings of the current study revealed that the diagnostic performance of CT-FFR was significantly impaired in lesions with more than a half-circle calcification, and lesion-specific calcium morphology was the only independent predictor of misclassified lesions by CT-FFR. Moreover, the excellent diagnostic performance of dynamic CT-MPI was preserved in different subgroups despite the impact of the calcium burden severity or other factors. Using lesion-specific calcium morphology to guide the appropriate use of CT-FFR and CT-MPI would have resulted in a $27.0 \%$ and $33.9 \%$ reduction in radiation dose and contrast medium consumption, respectively, without compromising diagnostic accuracy.

CT-FFR has been considered a valuable imaging approach for the noninvasive functional evaluation of coronary stenosis. Compared with CCTA, neither the CFD nor ML-based method has a higher diagnostic accuracy when invasive FFR is used as the reference standard $(9,12,23,24)$. However, a fundamental step in CT-FFR simulation is vessel segmentation and lumen delineation $(8,17)$, but the accuracy of this step could be impaired by the blooming artifact caused by severe calcification. Several previous studies have investigated the influence of vessel calcification on the diagnostic accuracy of CT-FFR (25-27). Their findings did not reveal a significantly different CTFFR performance across subgroups with a variety of patientspecific CACS, but this score only reflects the cumulative calcium score of all vessels; it does not necessarily pertain to the lesion-specific calcium burden and cannot precisely evaluate the true severity of the target lesion calcification. In addition, previous vessel-based analyses also demonstrated that the specificity of vessel-based CACS tended to decrease (25-27), which is consistent with our findings. Moreover, the morphology of the calcium ring also negatively impacts the visualization of the coronary lumen (28). This parameter has not been investigated in previous CT-FFR studies.

The current study shows that calcification morphology was the best parameter to discriminate between target lesions with correct and incorrect CT-FFR results. In cases with a calcium ring $>180^{\circ}$, diagnostic accuracy significantly decreased compared to lesions with a calcium ring $<180^{\circ}$. This could be attributed to the more prominent blooming artifact caused by a half-circle or circumferential calcification. When the vessel lumen was surrounded by severe calcification, CT-FFR was prone to overestimate the calcium volume and underestimate the lumen area. In such a circumstance, inaccurate vessel segmentation resulted in discrepancies between the CT-FFR and invasive FFR findings, more specifically, predominantly false-positive results due to CT-FFR underestimation of the lumen geometry. In contrast to calcium morphology, neither the vessel-based CACS nor patient-based CACS could differentiate matched lesions from unmatched lesions in CT-FFR results. This finding suggests that the appropriate use of CT-FFR should be guided by the lesion-specific calcium morphology assessment rather than the patientbased or vessel-based CACS.

In contrast to CT-FFR, dynamic CT-MPI evaluates the hemodynamic significance of coronary stenosis by absolute quantification of MBF, where the calculation is not affected by the anatomical features of the target lesion. In this study, the diagnostic performance of dynamic CT-MPI remained at a high level regardless of the impact of any imaging characteristics (calcium morphology, CACS, DS, or lesion length). Notably, dynamic CT-MPI outperformed CTFFR in functional evaluation across all subgroups. For lesions with a cross-sectional calcium deposition $>180^{\circ}$, this discrepancy was the most prominent, showing a diagnostic accuracy for dynamic CT-MPI and CT-FFR of $93.9 \%$ and $62.2 \%$, respectively. In other words, the clinical use of CTFFR should be replaced by dynamic CT-MPI when crosssectional calcification involves more than 2 quadrants.

In light of the above findings, lesion-specific calcium morphology may be the best parameter to optimize the clinical use of CT-based functional evaluations. When the target lesion shows more than a half-circle calcification, CT-FFR should be avoided as the specificity, NPV, and PPV were all significantly lower than those of dynamic CT-MPI. In this circumstance, dynamic CT-MPI is the preferred imaging method over CT-FFR for the precise assessment of the hemodynamic significance of coronary stenosis. On the other hand, for lesions with less than a half-circle calcification, the NPV of CT-FFR was equally high compared with dynamic CT-MPI, making CT-FFR a reliable approach to safely rule out flow-limiting stenosis in cases of noncalcified or mildly calcified lesions. In these cases, the extra contrast medium and radiation dose can be avoided if the CT-FFR shows a negative result and dynamic CT-MPI is not required. However, the PPVs of CT-FFR 
were low regardless of calcium morphology. Therefore, even when CT-FFR reveals a positive result, dynamic CTMPI is still warranted to reduce unnecessary downstream invasive tests (due to the many false-positive cases arising from CT-FFR). If the above diagnostic strategy had been applied to the current cohort, dynamic CT-MPI could have been avoided in $33.9 \%$ of patients while maintaining uncompromised diagnostic accuracy, leading to a $27.0 \%$ and $33.9 \%$ reduction in radiation dose and contrast medium usage, respectively. Furthermore, previous studies have demonstrated that CT-FFR may improve downstream clinical management in patients with an intermediateto-high pretest probability of CAD $(29,30)$. The current findings also confirmed the value of CT-FFR in guiding clinical decisions, especially when integrated with dynamic CT-MPI.

Despite the promising results, the current study has several limitations. First, dynamic CT-MPI was performed on a third-generation dual-source CT, and CTFFR simulation used an ML-based approach. These 2 techniques are not widely used in current clinical practice. Therefore, further studies using other CT-MPI and CTFFR techniques are needed to validate the generalizability of the present findings. Moreover, our ML-based CT-FFR model was trained using a synthetically generated database of coronary vasculature, which employed a CFD-based method rather than invasive FFR as the reference standard. The diagnostic performance of this approach might be suboptimal in a CFD-based simulation. In addition, the retrospective design of the current study had an inherent inclusion bias insofar as patients with a higher pretest probability and positive CT results were more likely to undergo downstream invasive tests. The present cohort may not represent the typical population referred for CCTA in clinical practice. Thus, future prospective studies are warranted to confirm that the current findings can be generalized to a population with a typical intermediate pretest probability of CAD.

In conclusion, the diagnostic performance of CT-FFR was significantly poorer for lesions with a cross-sectional calcium arc $>180^{\circ}$. Lesion-specific calcium morphology is the preferred parameter for guiding the appropriate use of CT-based functional assessments.

\section{Acknowledgments}

Funding: This study was supported by the Medical Guidance Scientific Research Support Project of Shanghai Science and Technology Commission (grant No. 19411965100) and the Shanghai Municipal Education Commission-Gaofeng Clinical Medicine Grant Support (grant No. 20161428).

\section{Footnote}

Reporting Checklist: The authors have completed the STARD reporting checklist. Available at https://dx.doi. org/10.21037/qims-21-491

Conflicts of Interest: All authors have completed the ICMJE uniform disclosure form (available at https://dx.doi. org/10.21037/qims-21-491). JZ reports grant support by the Medical Guidance Scientific Research Support Project of Shanghai Science and Technology Commission (grant No. 19411965100) and the Shanghai Municipal Education Commission-Gaofeng Clinical Medicine Grant Support (grant No. 20161428). The other authors have no conflicts of interest to declare.

Ethical Statement: The authors are accountable for all aspects of the work in ensuring that questions related to the accuracy or integrity of any part of the work are appropriately investigated and resolved. The study was conducted in accordance with the Declaration of Helsinki (as revised in 2013). The hospital ethics committee approved this retrospective study (No. 2020-91) and waived written informed consent from all patients.

Open Access Statement: This is an Open Access article distributed in accordance with the Creative Commons Attribution-NonCommercial-NoDerivs 4.0 International License (CC BY-NC-ND 4.0), which permits the noncommercial replication and distribution of the article with the strict proviso that no changes or edits are made and the original work is properly cited (including links to both the formal publication through the relevant DOI and the license). See: https://creativecommons.org/licenses/by-nc-nd/4.0/.

\section{References}

1. Knuuti J, Wijns W, Saraste A, Capodanno D, Barbato E, Funck-Brentano C, et al. 2019 ESC Guidelines for the diagnosis and management of chronic coronary syndromes. Eur Heart J 2020;41:407-77.

2. Xaplanteris P, Fournier S, Pijls NHJ, Fearon WF, Barbato E, Tonino PAL, et al. Five-Year Outcomes with PCI Guided by Fractional Flow Reserve. N Engl J Med 
2018;379:250-9.

3. Yu L, Tao X, Dai X, Liu T, Zhang J. Dynamic CT Myocardial Perfusion Imaging in Patients without Obstructive Coronary Artery Disease: Quantification of Myocardial Blood Flow according to Varied Heart Rate Increments after Stress. Korean J Radiol 2021;22:97-105.

4. Yu M, Shen C, Dai X, Lu Z, Wang Y, Lu B, Zhang J. Clinical Outcomes of Dynamic Computed Tomography Myocardial Perfusion Imaging Combined With Coronary Computed Tomography Angiography Versus Coronary Computed Tomography Angiography-Guided Strategy. Circ Cardiovasc Imaging 2020;13:e009775.

5. Yu M, Chen X, Dai X, Pan J, Wang Y, Lu B, Zhang J. The Value of Low-Dose Dynamic Myocardial Perfusion CT for Accurate Evaluation of Microvascular Obstruction in Patients With Acute Myocardial Infarction. AJR Am J Roentgenol 2019;213:798-806.

6. Ma H, Dai X, Yang X, Zhao X, Wang R, Zhang J. Clinical and imaging predictors of impaired myocardial perfusion in symptomatic patients after percutaneous coronary intervention: insights from dynamic CT myocardial perfusion imaging. Quant Imaging Med Surg 2021;11:3327-37.

7. Li Y, Yuan M, Yu M, Lu Z, Shen C, Wang Y, Lu B, Zhang J. Prevalence of Decreased Myocardial Blood Flow in Symptomatic Patients with Patent Coronary Stents: Insights from Low-Dose Dynamic CT Myocardial Perfusion Imaging. Korean J Radiol 2019;20:621-30.

8. Taylor CA, Fonte TA, Min JK. Computational fluid dynamics applied to cardiac computed tomography for noninvasive quantification of fractional flow reserve: scientific basis. J Am Coll Cardiol 2013;61:2233-41.

9. Nørgaard BL, Leipsic J, Gaur S, Seneviratne S, Ko BS, Ito $\mathrm{H}$, et al. Diagnostic performance of noninvasive fractional flow reserve derived from coronary computed tomography angiography in suspected coronary artery disease: the NXT trial (Analysis of Coronary Blood Flow Using CT Angiography: Next Steps). J Am Coll Cardiol 2014;63:1145-55.

10. Li Y, Yu M, Dai X, Lu Z, Shen C, Wang Y, Lu B, Zhang J. Detection of Hemodynamically Significant Coronary Stenosis: CT Myocardial Perfusion versus Machine Learning CT Fractional Flow Reserve. Radiology 2019;293:305-14.

11. Li Y, Dai X, Lu Z, Shen C, Zhang J. Diagnostic performance of quantitative, semi-quantitative, and visual analysis of dynamic CT myocardial perfusion imaging: a validation study with invasive fractional flow reserve. Eur
Radiol 2021;31:525-34.

12. Min JK, Leipsic J, Pencina MJ, Berman DS, Koo BK, van Mieghem C, Erglis A, Lin FY, Dunning AM, Apruzzese P, Budoff MJ, Cole JH, Jaffer FA, Leon MB, Malpeso J, Mancini GB, Park SJ, Schwartz RS, Shaw LJ, Mauri L. Diagnostic accuracy of fractional flow reserve from anatomic CT angiography. JAMA 2012;308:1237-45.

13. Yu M, Li Y, Li W, Lu Z, Wei M, Zhang J. Calcification Remodeling Index Characterized by Cardiac CT as a Novel Parameter to Predict the Use of Rotational Atherectomy for Coronary Intervention of Lesions with Moderate to Severe Calcification. Korean J Radiol 2017;18:753-62.

14. Cury RC, Abbara S, Achenbach S, Agatston A, Berman DS, Budoff MJ, Dill KE, Jacobs JE, Maroules CD, Rubin GD, Rybicki FJ, Schoepf UJ, Shaw LJ, Stillman AE, White CS, Woodard PK, Leipsic JA. CAD-RADS ${ }^{\mathrm{TM}}$ : Coronary Artery Disease - Reporting and Data System: An Expert Consensus Document of the Society of Cardiovascular Computed Tomography (SCCT), the American College of Radiology (ACR) and the North American Society for Cardiovascular Imaging (NASCI). Endorsed by the American College of Cardiology. J Am Coll Radiol 2016;13:1458-1466.e9.

15. Bamberg F, Klotz E, Flohr T, Becker A, Becker CR, Schmidt B, Wintersperger BJ, Reiser MF, Nikolaou K. Dynamic myocardial stress perfusion imaging using fast dual-source CT with alternating table positions: initial experience. Eur Radiol 2010;20:1168-73.

16. Cerqueira MD, Weissman NJ, Dilsizian V, Jacobs AK, Kaul S, Laskey WK, Pennell DJ, Rumberger JA, Ryan T, Verani MS; American Heart Association Writing Group on Myocardial Segmentation and Registration for Cardiac Imaging. Standardized myocardial segmentation and nomenclature for tomographic imaging of the heart. A statement for healthcare professionals from the Cardiac Imaging Committee of the Council on Clinical Cardiology of the American Heart Association. Circulation 2002;105:539-42.

17. Itu L, Rapaka S, Passerini T, Georgescu B, Schwemmer C, Schoebinger M, Flohr T, Sharma P, Comaniciu D. A machine-learning approach for computation of fractional flow reserve from coronary computed tomography. J Appl Physiol (1985) 2016;121:42-52.

18. Pijls NH, De Bruyne B, Peels K, Van Der Voort PH, Bonnier HJ, Bartunek J Koolen JJ, Koolen JJ. Measurement of fractional flow reserve to assess the functional severity of coronary-artery stenoses. N Engl J 
Med 1996;334:1703-8.

19. Nallamothu BK, Spertus JA, Lansky AJ, Cohen DJ, Jones PG, Kureshi F, et al. Comparison of clinical interpretation with visual assessment and quantitative coronary angiography in patients undergoing percutaneous coronary intervention in contemporary practice: the Assessing Angiography (A2) project. Circulation 2013;127:1793-800.

20. Tonino PA, Fearon WF, De Bruyne B, Oldroyd KG, Leesar MA, Ver Lee PN, Maccarthy PA, Van't Veer M, Pijls NH. Angiographic versus functional severity of coronary artery stenoses in the FAME study fractional flow reserve versus angiography in multivessel evaluation. J Am Coll Cardiol 2010;55:2816-21.

21. DeLong ER, DeLong DM, Clarke-Pearson DL. Comparing the areas under two or more correlated receiver operating characteristic curves: a nonparametric approach. Biometrics 1988;44:837-45.

22. Pencina MJ, D'Agostino RB Sr, Steyerberg EW. Extensions of net reclassification improvement calculations to measure usefulness of new biomarkers. Stat Med 2011;30:11-21.

23. Coenen A, Kim YH, Kruk M, Tesche C, De Geer J, Kurata A, Lubbers ML, Daemen J, Itu L, Rapaka S, Sharma P, Schwemmer C, Persson A, Schoepf UJ, Kepka C, Hyun Yang D, Nieman K. Diagnostic Accuracy of a MachineLearning Approach to Coronary Computed Tomographic Angiography-Based Fractional Flow Reserve: Result From the MACHINE Consortium. Circ Cardiovasc Imaging 2018;11:e007217.

24. Tesche C, De Cecco CN, Baumann S, Renker M, McLaurin TW, Duguay TM, Bayer RR 2nd, Steinberg DH, Grant KL, Canstein C, Schwemmer C, Schoebinger M, Itu LM, Rapaka S, Sharma P, Schoepf UJ. Coronary CT Angiography-derived Fractional Flow Reserve: Machine Learning Algorithm versus Computational Fluid Dynamics Modeling. Radiology 2018;288:64-72.

Cite this article as: Dai X, Lu Z, Yu Y, Yu L, Xu H, Zhang J. The use of lesion-specific calcium morphology to guide the appropriate use of dynamic CT myocardial perfusion imaging and CT fractional flow reserve. Quant Imaging Med Surg 2022;12(2):1257-1269. doi: 10.21037/qims-21-491
25. Nørgaard BL, Gaur S, Leipsic J, Ito H, Miyoshi T, Park SJ, Zvaigzne L, Tzemos N, Jensen JM, Hansson N, Ko B, Bezerra H, Christiansen EH, Kaltoft A, Lassen JF, Bøtker $\mathrm{HE}$, Achenbach S. Influence of Coronary Calcification on the Diagnostic Performance of CT Angiography Derived FFR in Coronary Artery Disease: A Substudy of the NXT Trial. JACC Cardiovasc Imaging 2015;8:1045-55.

26. Tesche C, Otani K, De Cecco CN, Coenen A, De Geer J, Kruk M, Kim YH, Albrecht MH, Baumann S, Renker M, Bayer RR, Duguay TM, Litwin SE, Varga-Szemes A, Steinberg DH, Yang DH, Kepka C, Persson A, Nieman K, Schoepf UJ. Influence of Coronary Calcium on Diagnostic Performance of Machine Learning CT-FFR: Results From MACHINE Registry. JACC Cardiovasc Imaging 2020;13:760-70.

27. Tang CX, Liu CY, Lu MJ, Schoepf UJ, Tesche C, Bayer RR 2nd, et al. CT FFR for Ischemia-Specific CAD With a New Computational Fluid Dynamics Algorithm: A Chinese Multicenter Study. JACC Cardiovasc Imaging 2020;13:980-90.

28. Yu M, Li Y, Li W, Lu Z, Wei M, Zhang J. Calcification remodeling index assessed by cardiac CT predicts severe coronary stenosis in lesions with moderate to severe calcification. J Cardiovasc Comput Tomogr 2018;12:42-9.

29. Nous FMA, Budde RPJ, Lubbers MM, Yamasaki Y, Kardys I, Bruning TA, Akkerhuis JM, Kofflard MJM, Kietselaer B, Galema TW, Nieman K. Impact of machine-learning CT-derived fractional flow reserve for the diagnosis and management of coronary artery disease in the randomized CRESCENT trials. Eur Radiol 2020;30:3692-701.

30. Lu MT, Ferencik M, Roberts RS, Lee KL, Ivanov A, Adami E, Mark DB, Jaffer FA, Leipsic JA, Douglas PS, Hoffmann U. Noninvasive FFR Derived From Coronary CT Angiography: Management and Outcomes in the PROMISE Trial. JACC Cardiovasc Imaging 2017;10:1350-8. 


\section{Appendix 1}

\section{Methods}

\section{CT acquisition and reconstruction}

A third-generation dual source CT (SOMATOM Force, Siemens Healthineers) was employed for CT-MPI and CCTA imaging.

The scan range of dynamic CT-MPI will be planned based on the calcium score images to cover the whole left ventricle. Intravenous ATP infusion will be maintained for 3 minutes at $160 \mu \mathrm{g} / \mathrm{kg} / \mathrm{min}$ before CT-MPI scan. A bolus of contrast media (50 mL, Iopamidol, $370 \mathrm{mg}$ iodine/mL, Bayer, Germany) will be injected into antecubital vein at the rate of $6 \mathrm{~mL} / \mathrm{s}$, followed by a $40 \mathrm{~mL}$ saline flush. Dynamic CT-MPI acquisition will be started 4 seconds after the begin of contrast injection. The end-systolic phase (triggered at $250 \mathrm{~ms}$ after the $\mathrm{R}$ wave in all participants) is set for the dynamic acquisition by using a shuttle mode technique with a coverage of $10.5 \mathrm{~cm}$ for complete imaging of the whole left ventricle. Scans will be launched every second or third heart cycle according to participants' heart rate, resulting in a series of 10 to 15 phases acquired over a fixed period of $32 \mathrm{~s}$. The acquisition parameters of dynamic CT-MPI are listed as follow: collimation $=96 \times 0.6 \mathrm{~mm}^{2}$, CARE $\mathrm{kV}$ will be used and the reference tube voltage $=80 \mathrm{kVp}$, rotation time $=250 \mathrm{~ms}$, CARE dose $4 \mathrm{D}$ will be used and the effective current $=300 \mathrm{mAs}$, reconstructed slice thickness $=3 \mathrm{~mm}$ and reconstructed slice interval $=2 \mathrm{~mm}$.

A bolus of contrast media (iopamidol $370 \mathrm{mg}$ iodine/mL, Bayer, Germany) will be injected into the antecubital vein at the rate of $4.5-5 \mathrm{~mL} / \mathrm{s}$, followed by injection of a $40 \mathrm{~mL}$ saline flush, using a dual-barrel power injector (Tyco-Mallinckrodt, US). The amount of contrast media will be determined according to the patient's body weight and the scanning time. Prospective ECG-triggered sequential acquisition will be performed in all participants for CCTA, with triggering window coveting from end-systolic to mid-diastolic phase. The detailed parameters are listed as follow: collimation $=96 \times 0.6 \mathrm{~mm}^{2}$, reconstructed slice thickness $=0.75 \mathrm{~mm}$, reconstructed slice interval $=0.5 \mathrm{~mm}$, rotation time $=250 \mathrm{~ms}$ and application of automated tube voltage and current modulation (CAREKv, CAREDose 4D, Siemens Healthineers, Germany). The reference tube current will be set as $320 \mathrm{mAs}$ and the reference tube voltage will be set as $100 \mathrm{kVp}$.

\section{CT-FFR measurement}

As introduced recently, we used a machine-learning based algorithm for CT-FFR simulation (cFFR, version 3.0, Siemens Healthineers). It's an alternative to physics-based approach and can be used on-site to calculate CT-FFR value. It's trained using a synthetically generated database of 12,000 different anatomies of coronary arteries with randomly placed stenosis among different branches and bifurcations. A computational fluid dynamics (CFD) by solving reduced-ordered NavierStokes equations is applied to calculate the pressure and flow distribution for each coronary tree. Quantitative features of anatomy and computed CT-FFR value were extracted for each location along the coronary tree. Then deep machine learning model is trained by using a deep neural network with four hidden layers to learn the relationship between the FFR value and quantitative anatomic features.

For the on-site processing, after CCTA data were successfully loaded, the centerline and luminal contours for whole coronary tree were automatically generated. The centerline and luminal contour are fundamental and critical information for computing CT-FFR value. They were manually adjusted when needed. Users then manually identified all stenotic lesions to extract their geometrical features required for cFFR algorithm. Finally, those data were input into the pre-learned model and cFFR was computed automatically at all locations in the coronary arterial tree, and the resulting values were visualized by color-coded 3D coronary maps. 

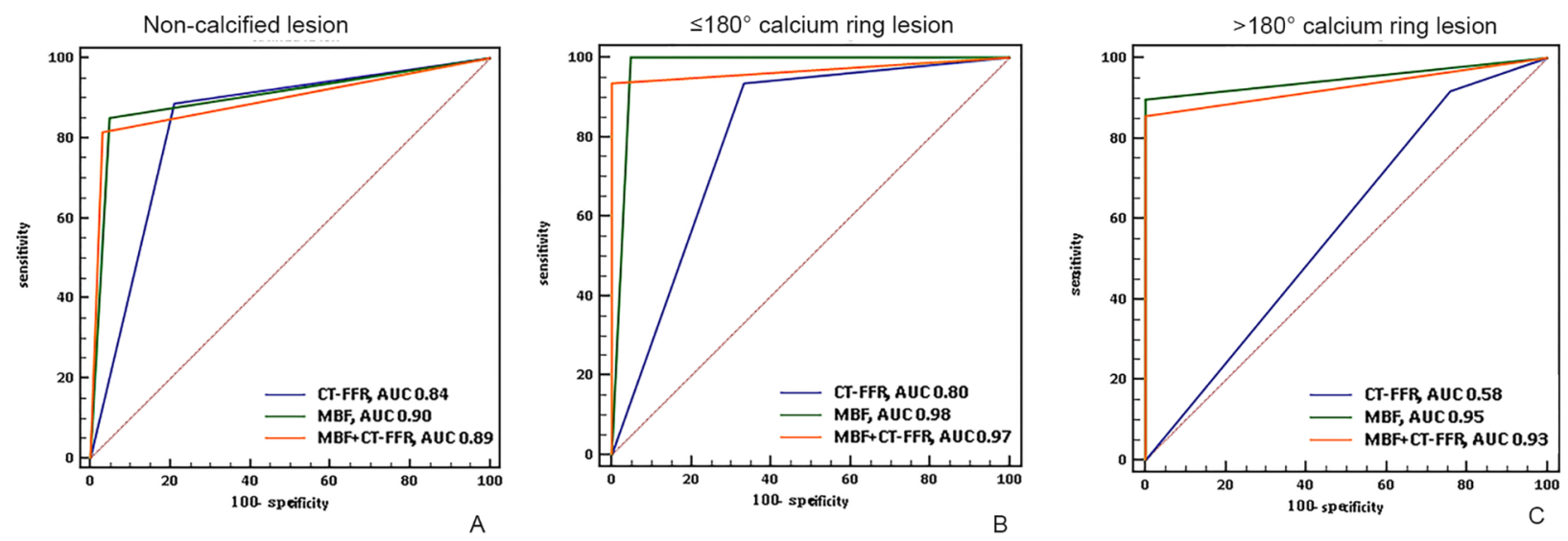

Figure S1 ROC curve analysis of dynamic CT-MPI, CT-FFR and CT-FFR + dynamic CT-MPI for identifying functionally significant coronary stenosis according to calcium morphology with reference to ICA/FFR. (A) For non-calcified lesions, the AUCs of CT-FFR, dynamic CT-MPI and CT-FFR + dynamic CT-MPI were similar between subgroups (all P>0.05). (B) For calcified lesions with calcium arc $\leq 180^{\circ}$, the AUCs of dynamic CT-MPI and CT-FFR + dynamic CT-MPI were significantly larger than that of CT-FFR (all P<0.01). (C) For calcified lesions with calcium arc $>180^{\circ}$, the AUCs of dynamic CT-MPI and CT-FFR + dynamic CT-MPI were significantly larger than that of CT-FFR (all P<0.001). AUC, area under curve; CT, computed tomography; CT-MPI, computed tomography myocardial perfusion imaging; FFR, fractional flow reserve; ICA, invasive coronary angiography; MBF, myocardial blood flow; ROC, receiver operating characteristic.

Table S1 Interobserver reproducibility

\begin{tabular}{|c|c|c|c|c|}
\hline & ICC & Kappa & $95 \% \mathrm{Cl}$ & $P$ value \\
\hline Diameter stenosis & 0.889 & - & $0.816-0.945$ & $<0.001$ \\
\hline Lesion length & 0.973 & - & $0.950-0.986$ & $<0.001$ \\
\hline CT-FFR & 0.886 & - & $0.776-0.942$ & $<0.001$ \\
\hline Calcium morphology & - & 0.884 & - & $<0.001$ \\
\hline
\end{tabular}

CACS, coronary artery calcium score; $\mathrm{Cl}$, Confidence interval; CT, computed tomography; FFR, fractional flow reserve; ICC, intraclass correlation coefficient; MBF, myocardial blood flow.

Table S2 Intra-observer reproducibility

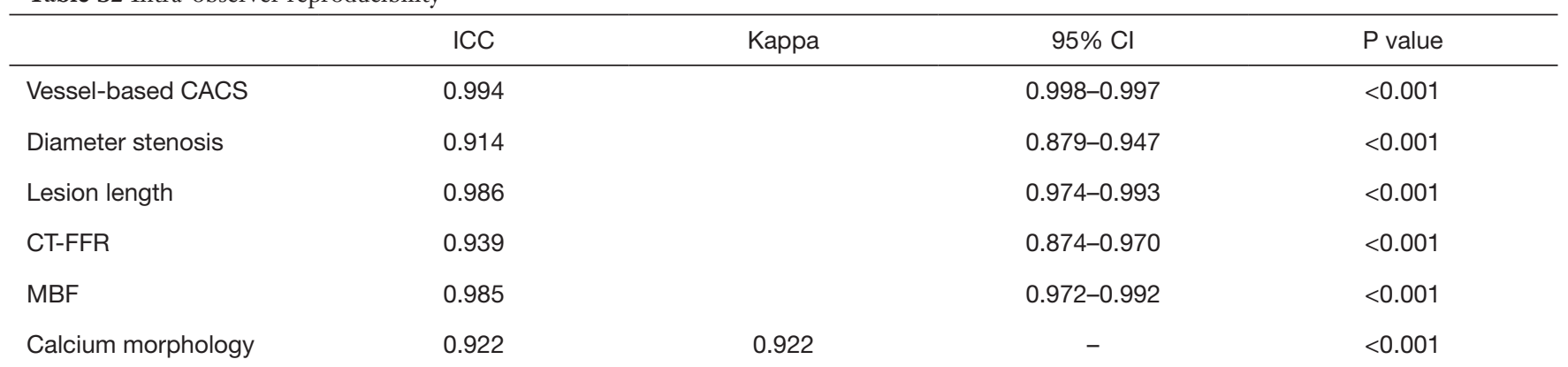

CACS, coronary artery calcium score; Cl, Confidence interval; CT, computed tomography; FFR, fractional flow reserve; ICC, intraclass correlation coefficient; MBF, myocardial blood flow. 
Table S3 Univariable and multivariable analyses of MBF for mismatch with ICA/FFR

\begin{tabular}{|c|c|c|c|c|c|c|}
\hline Characteristics & \multicolumn{3}{|c|}{ Univariable analysis } & \multicolumn{3}{|c|}{ Multivariable analysis } \\
\hline Vessel-based CACS & 0.999 & $0.997-1.001$ & 0.556 & 1.000 & $0.997-1.003$ & 0.937 \\
\hline Diameter stenosis & 1.181 & $0.845-1.651$ & 0.329 & 1.269 & $0.888-1.814$ & 0.191 \\
\hline Lesion length & 1.000 & $0.971-1.031$ & 0.984 & - & - & - \\
\hline Number of calcified segments of target vessels & 0.682 & $0.386-1.206$ & 0.189 & 0.513 & $0.198-1.327$ & 0.169 \\
\hline
\end{tabular}

CACS, coronary artery calcium score; Cl, Confidence interval; FFR, fractional flow reserve; ICA, invasive coronary angiography; MBF, myocardial blood flow.

Table S4 Vessel-based diagnostic performance of CT-FFR and MBF according to vessel-specific CACS

\begin{tabular}{|c|c|c|c|c|c|c|c|c|c|c|c|c|}
\hline & \multicolumn{3}{|c|}{ Q1 $(n=57)$} & \multicolumn{3}{|c|}{ Q2 $(n=58)$} & \multicolumn{3}{|c|}{ Q3 $(n=57)$} & \multicolumn{3}{|c|}{ Q4 $(n=57)$} \\
\hline & $\mathrm{MBF}^{\star}$ & CT-FFR ${ }^{\S}$ & $P$ value & MBF & CT-FFR & $P$ value & MBF & CT-FFR & $P$ value & MBF & CT-FFR & $P$ value \\
\hline Sensitivity & $76.9(10 / 13)$ & $84.6(11 / 13)$ & 1.000 & $89.5(17 / 19)$ & $84.2(16 / 19)$ & 1.000 & $93.1(27 / 29)$ & $93.1(27 / 29)$ & 1.000 & $93.5(29 / 31)$ & $96.8(30 / 31)$ & 1.000 \\
\hline \multirow[t]{2}{*}{ Specificity } & $97.7(43 / 44)$ & $81.8(36 / 44)$ & 0.016 & $92.3(36 / 39)$ & $59.0(23 / 39)$ & 0.002 & $100(28 / 28)$ & $57.1(16 / 28)$ & $<0.001$ & $96.2(25 / 26)$ & $38.5(10 / 26)$ & $<0.001$ \\
\hline & [88.0-99.9] & [67.3-91.8] & & [79.1-98.4] & [42.1-74.4] & & [87.7-100.0] & {$[37.2-75.5]$} & & [80.4-99.9] & [20.2-59.4] & \\
\hline \multirow[t]{2}{*}{ PPV } & $90.9(10 / 11)$ & $57.9(11 / 19)$ & 0.100 & $85.0(17 / 20)$ & $50.0(16 / 32)$ & 0.011 & $100(27 / 27)$ & $69.2(27 / 39)$ & 0.001 & $96.7(29 / 30)$ & $65.2(30 / 46)$ & 0.001 \\
\hline & [58.5-98.6] & [41.3-72.8] & & [65.4-94.4] & [39.6-60.4] & & - & {$[59.2-77.7]$} & & [80.9-99.5] & [57.8-71.9] & \\
\hline \multirow[t]{2}{*}{ Accuracy } & $93.0(53 / 57)$ & $82.5(47 / 57)$ & 0.008 & $91.4(53 / 58)$ & $67.2(39 / 58)$ & 0.012 & $96.5(55 / 57)$ & $75.4(43 / 57)$ & 0.002 & $94.7(54 / 57)$ & $70.1(40 / 57)$ & $<0.001$ \\
\hline & [83.0-98.1] & [70.1-91.3] & & [81.0-97.1] & [53.7-79.0] & & [87.9-99.6] & [62.2-85.9] & & [85.4-98.9] & [56.6-81.6] & \\
\hline
\end{tabular}

Group by quartile. *, MBF cut-off value $=99 \mathrm{~mL} / 100 \mathrm{~mL} / \mathrm{min} ;{ }^{\S}$, CT-FFR cut-off value $=0.78$. CT, computed tomography; FFR, fractional flow reserve; MBF, myocardial blood flow; CACS, coronary artery calcium score; NPV, negative predictive value; PPV, positive predictive value.

Table S5 Patient-based diagnostic performance of CT-FFR and MBF according to CAD-RADS grade

\begin{tabular}{|c|c|c|c|c|c|c|}
\hline & \multicolumn{3}{|c|}{ CAD-RADS 2 and $3(n=60)$} & \multicolumn{3}{|c|}{ CAD-RADS $4(n=120)$} \\
\hline Sensitivity & $\begin{array}{c}87.5(7 / 8) \\
{[47.4-99.7]}\end{array}$ & $\begin{array}{c}75.0(6 / 8) \\
{[34.9-96.8]}\end{array}$ & 1.000 & $\begin{array}{c}90.0(63 / 70) \\
{[80.5-95.9]}\end{array}$ & $\begin{array}{c}88.6(62 / 70) \\
{[78.7-94.93]}\end{array}$ & 1.000 \\
\hline Specificity & $\begin{array}{l}100(52 / 52) \\
{[93.2-100]}\end{array}$ & $\begin{array}{c}78.8(41 / 52) \\
{[65.3-88.9]}\end{array}$ & $<0.001$ & $\begin{array}{c}94.0(47 / 50) \\
{[83.5-98.8]}\end{array}$ & $\begin{array}{c}58.0(29 / 50) \\
{[43.2-71.8]}\end{array}$ & $<0.001$ \\
\hline PPV & $\begin{array}{c}100(7 / 7) \\
-\end{array}$ & $\begin{array}{l}35.3(6 / 17) \\
{[22.0-51.3]}\end{array}$ & 0.006 & $\begin{array}{c}95.5(63 / 66) \\
{[87.5-98.4]}\end{array}$ & $\begin{array}{c}74.7(62 / 83) \\
{[67.8-80.5]}\end{array}$ & 0.001 \\
\hline Accuracy & $\begin{array}{c}98.3(59 / 60) \\
{[91.1-100]}\end{array}$ & $\begin{array}{c}78.3(47 / 60) \\
{[65.8-87.9]}\end{array}$ & $<0.001$ & $\begin{array}{c}91.7(110 / 120) \\
{[85.2-95.9]}\end{array}$ & $\begin{array}{c}75.8(91 / 120) \\
{[67.2-83.2]}\end{array}$ & $<0.001$ \\
\hline
\end{tabular}

*, MBF cut-off value $=99 \mathrm{~mL} / 100 \mathrm{~mL} / \mathrm{min} ;{ }^{\S}$, CT-FFR cut-off value $=0.76$. CAD-RADS, Coronary Artery Disease-Reporting and Data System; CT, computed tomography; FFR, fractional flow reserve; MBF, myocardial blood flow; CACS, coronary artery calcium score; NPV, negative predictive value; PPV, positive predictive value. 